\title{
Para além dos dados coletados: Políticas das APIs nas plataformas de mídias digitais ${ }^{a}$ Beyond data collection: Politics of API's on social media platforms
}

\author{
CARLOS D'A NDRÉ A b \\ Universidade Federal de Minas Gerais, Programa de Pós-Graduação em Comunicação. Belo \\ Horizonte - MG, Brasil
}

\section{RESUMO}

O artigo discute questões conceituais e metodológicas a serem enfrentadas por pesquisas empíricas baseadas em dados obtidos através de Application Programming Interfaces (APIs) de plataformas online. Em diálogo com os Estudos de Plataforma, métodos digitais e outras abordagens ancoradas nos estudos de ciência e tecnologia (STS), o artigo volta-se para a relação entre as políticas das APIs e dimensões como affordances, governança, datificação e mediações algorítmicas em mídias sociais como Twitter e Facebook. Aspectos materiais, políticos, normativos e econômicos são discutidos por meio de exemplos que vão da implementação das primeiras APIs, nos anos 2000, aos desafios recentes, em especial após o escândalo Cambridge Analytica.

Palavras-chave: Plataforma de mídia social, métodos digitais, datificação, algoritmos, APIs

${ }^{a}$ Este trabalho é parte de pesquisa financiada pelo CNPq (edital Universal 2016 407462/2016) e CAPES (estágio pós-doutoral no exterior).

${ }^{\mathrm{b}}$ Professor do Programa de Pós-Graduação em Comunicação (PPGCOM) da Universidade Federal de Minas Gerais (UFMG). Coordenador do grupo de pesquisa R-EST - estudos redes sociotécnicas. Orcid: https://orcid.org/00000001-7328-6714. E-mail: carlosfbd@gmail.com

\begin{abstract}
This article discusses the conceptual and methodological issues faced during empirical research based on data collected via Application Programming Interfaces (APIs) of online platforms. Based on Platform Studies, digital methods, and other approaches anchored in Science and Technology Studies (STS), this article examines the entanglements between the politics of APIs and dimensions, such as affordances, governance, datafication, and algorithmic mediations in social media platforms (e.g., Twitter and Facebook). Material, political, normative, and economic aspects are discussed by examples ranging from the first APIs implemented in the 2000s to recent challenges, especially those following the Cambridge Analytica scandal.

Keywords: Social media platform, digital methods, datafication, algorithms, APIs
\end{abstract}


${ }^{1}$ Em alinhamento como autores como Bruns (2019), neste artigo adotamos o termo "API pública" para designar os recursos que permitem acessos não pagos a dados fornecidos por plataformas privadas.

${ }^{2}$ No original: "provide information to third-party applications through 'calls', a technique of retrieving data on a server in the background, without disrupting the display and function of a web page". Esta e demais traduções, do autor.

${ }^{3}$ No original: "a type of investigation based on the information collected by social media platforms and made available through standardized commands to query, filter, format and download such information".

\section{INTRODUÇÃO}

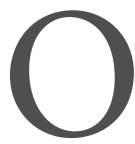

ACESSO PARCIAL À grande quantidade de rastros armazenados por plataformas como Facebook, Twitter e YouTube e a diversidade de aplicações que permitem a extração, o processamento e a visualização de dados contribuíram, desde o fim da década de 2010, para um significativo crescimento do uso, nas pesquisas acadêmicas e de mercado, de dados fornecidos por mídias sociais. Por meio de suas Application Programming Interfaces (APIs), plataformas online oferecem acesso a uma parte dos dados por elas gerados e/ou coletados, como os gêneros musicais atribuídos a um artista (Spotify), as postagens vinculadas a um termo ou a uma hashtag (Twitter) ou o total de visualizações, curtidas e comentários, até um dado momento, dos vídeos postados por um canal (YouTube). Já plataformas como Instagram (em 2016) e Facebook (em 2018) cercearam o acesso às suas APIs públicas ${ }^{1}$, tornando ainda mais evidentes os atravessamentos políticos e epistemológicos das políticas de dados das plataformas discutidos anteriormente por autores como Bucher (2013) e Puschmann e Burgess (2014).

Ao padronizar os procedimentos de acesso e troca de informações entre sistemas computacionais, explica Bodle (2011), uma APIs aberta "provê informações a aplicativos de terceiros através de 'requisições', uma técnica de recuperação de dados em um servidor sem que se interrompa a exibição e o funcionamento de uma página web”2 (p. 322). A adoção generalizada de APIs por serviços web ao longo dos anos 2000 é, segundo Helmond (2015), o principal pilar do processo de "plataformização da web", isto é, da consolidação das plataformas online como um modelo infraestrutural e econômico que, por basear-se no intercâmbio controlado de dados, se opõe ao modelo mais descentralizado que orientara a World Wide Web nos anos 1990.

O acesso facilitado a dados via APIs públicas pode ser tomado como um marco para o avanço no uso de "métodos computacionais" (Vimieiro \& Bargas, 2019) por pesquisadores interessados nas dinâmicas das plataformas de mídia social. Nesse contexto, Venturini e Rogers (2019) identificam a emergência de um conjunto de "pesquisas baseadas em APIs", definida como "um tipo de investigação baseada nas informações coletadas pelas plataformas de mídia social e disponibilizadas por meio de comandos padronizados para consultar, filtrar, formatar e baixar essas informações"3 (pp. 1-2).

Pode-se dizer que a popularização de ferramentas e de procedimentos a partir das possibilidades de uso das APIs abriu frentes muito promissoras de pesquisa para as ciências sociais e para as humanidades, principalmente em diálogo com outras áreas do conhecimento, como a ciência da computação e o design (visualização de dados). Por outro lado, a relativa facilidade de extração 
de dados e o alívio de ter logo em mãos a coleta de dados relativos a um tema resulta, frequentemente, na invisibilidade das mediações tecnológicas, políticas e econômicas exercidas pelas plataformas online.

Visando contribuir para abordagens que tensionem leituras positivistas baseadas em uma crença na objetividade dos dados (van Dijck, 2017), assumimos neste trabalho que as APIs são "arranjos contingentes históricos de componentes sociais e materiais que se combinam para produzir novas realidades"4 (Bucher, 2013, p. 1). Assim, argumentamos que o acesso facilitado a um conjunto revelador de dados das mídias sociais não pode ser dissociado do modo como as APIs, ao produzirem e induzirem leituras e interpretações, conformam tanto nossa forma de compreender as plataformas como as temáticas que nela se desenrolam. A abordagem de Bucher (2013) e de parte significativa da bibliografia aqui mobilizada se ancora principalmente nos Estudos de Ciência e Tecnologia (em inglês, Science and Technology Studies, ou STS), um campo transdisciplinar que tem como uma de suas questões centrais a compreensão do modus operandi dos objetos técnicos a partir das condições políticas e epistemológicas em que suas materialidades são constituídas e apropriadas (Law, 2017).

Retomando discussões de trabalhos anteriores - em especial d'Andréa (2018) -, no presente artigo nos voltamos para algumas questões conceituais e metodológicas a serem enfrentadas por pesquisas empíricas baseadas em dados fornecidos por plataformas de mídia social. Mais especificamente, discutimos como os estudos baseados em dados obtidos via APIs podem tensionar as lógicas de datificação incorporadas pelas plataformas, principalmente através da normatização das ações dos usuários, das gestões políticas e econômicas das APIs e do modo como os dados são hierarquizados por algoritmos de recomendação. Cientes dos riscos de perdermos especificidades, neste trabalho não nos voltaremos para uma APIs ou para uma plataforma específica. Em diálogo com autores que se dedicaram a cuidadosos estudos de caso, nossa proposta é oferecer um conjunto de reflexões e apontamentos baseados em características e exemplos de diferentes mídias sociais, com certo destaque para o Facebook e o Twitter.

Iniciamos o artigo apresentando os Estudos de Plataforma, que reúnem um conjunto de conceitos, reflexões e práticas metodológicas que buscam compreender as articulações das dimensões técnicas, políticas e econômicas que constituem as mídias sociais e outras plataformas online (van Dijck et al., 2018). Principalmente a partir das contribuições dos pesquisadores vinculados à Escola de Amsterdã (Gorwa, 2019) ${ }^{5}$, estes estudos se ancoram no campo STS para discutir como Facebook, Uber e tantos outros serviços online se constituem a partir de dimensões como infraestrutura, mecanismos de governança, modelos de negócio e dos usos possíveis de suas materialidades (affordances)
${ }^{4}$ No original: "contingent arrangements of social and material components that coalesce to produce new realities".

${ }^{5} \mathrm{O}$ autor se refere a um conjunto de pesquisadores vinculados ao departamento de Media Studies da Universidade de Amsterdã (UvA), parte dos quais atua ainda no projeto Digital Methods Initiative (DMI). Cabe apontar, no entanto, que importantes pesquisadores holandeses estão vinculados a outras universidades, como a Utrecht University. 
(d'Andréa, 2020). Após situar os Estudos de Plataforma, na primeira seção do artigo apresentamos também algumas vertentes metodológicas que visam explorar as singularidades dos ambientes online. A partir do enfoque dos métodos digitais e de abordagens afins, apontamos para uma perspectiva reflexiva que tensiona a lógica (comercial, sobretudo) das plataformas online e sinalizamos caminhos para os estudos críticos baseados em dados fornecidos pelas APIs.

Na seção "(Política das) APIs: Primeiras Iniciativas”, apresentamos como a adoção generalizada das APIs culminou, ao longo dos anos 2000, no processo de plataformização da web, descrito por Helmond (2015). Elencamos as características técnicas das APIs e situamos as tensões políticas da época em torno da regulação da internet. A partir de um breve resgate histórico, apontamos ainda como as APIs se interligam e dão sustentação à ideologia da chamada web 2.0.

Em “Affordances, Governança, Algoritmos", voltamo-nos para esses três aspectos pertinentes às pesquisas baseadas em dados de plataformas de mídia social. Em primeiro lugar, sinalizamos a importância de compreender e desnaturalizar o modo como as plataformas mensuram e tornam disponíveis as práticas que abrigam. Funcionalidades como "curtir" ou "compartilhar" permitem às plataformas padronizar as atividades dos usuários, resultando em um processo de "gramatização da ação" (Gerlitz \& Rieder, 2018). Essa normatização das práticas orienta a oferta de dados via APIs, induzindo interpretações e análises fortemente alinhadas às lógicas comerciais de popularidade e de engajamento.

O segundo aspecto abordado chama a atenção para intrínseca relação entre a governança das APIs e as questões jurídicas e econômicas que perpassam a atuação das plataformas. $\mathrm{O}$ escândalo envolvendo a empresa Cambridge Analytica e o Facebook é brevemente retomado para situar um processo recente que, dentre outros desdobramentos, alavancou uma "crise nas pesquisas sobre mídias sociais"6 (Rogers, 2018b, p. 558). A crescente restrição e desativação de APIs públicas e o surgimento de iniciativas institucionais que visam direcionar as pesquisas (como o Social Science One, do Facebook) são alguns dos tópicos abordados.

O terceiro aspecto volta-se para a indissociabilidade entre os processos de datificação adotados pelas plataformas e as mediações algorítmicas que, ao performatizarem os dados, estabelecem lógicas personalizadas de seleção e de ranqueamento. Ao instaurar regimes próprios de conhecimento e de visibilidade, os algoritmos diversificam as experiências possíveis dos diferentes usuários de uma mídia social, o que coloca em xeque a representatividade muitas vezes atribuída por pesquisadores aos dados obtidos por meio das APIs. Esta questão mostra-se ainda mais delicada se considerada a acelerada adesão a técnicas de aprendizagem de máquina, como discute Mackenzie (2018). 
Visando, mais uma vez, dialogar com pesquisadores interessados em uma leitura crítica dos dados ofertados pelas plataformas, nas considerações finais retomamos os argumentos centrais do artigo para discutir a pertinência e os desafios das pesquisas baseadas em APIs.

\section{PLATAFORMAS ONLINE: DIMENSÕES E MÉTODOS}

Em obra que visa traçar um panorama da denominada "sociedade da plataforma", van Dijck et al. (2018) afirmam que "uma plataforma é alimentada com dados, automatizada e organizada por meio de algoritmos e interfaces, formalizada por meio de relações de propriedade orientadas por modelos de negócios e regidas por acordos de usuários"7 (p. 9). Mais do que uma definição, esta afirmação enfatiza como as agências de um extenso e heterogêneo conjunto de serviços on-line se organizam a partir da articulação de aspectos computacionais, normativos e econômicos.

Em elaboração desde o início dos anos 2010 por autores como Gillespie (2010) e van Dijck (2013), os Estudos de Plataforma compõem um construto teórico e metodológico central para compreendermos uma recente virada crítica nos estudos em internet. Antes baseadas em conceitos como colaboração, inteligência coletiva e web 2.0, as pesquisas atuais sobre mídias sociais e outras plataformas online assumem o desafio de compreendê-las não só como ambientes de interações, mas também a partir de sua robusta materialidade e de sua centralidade no regime capitalista contemporâneo.

Para entender esta perspectiva, como aponta Recuero (2019), é fundamental inclusive reconhecer as diferenças entre termos como plataforma, site de rede social ou simplesmente rede social. Um aspecto que consolida e singulariza a ideia de plataforma online é o reconhecimento de que a sociabilidade online emerge em articulação com robustas infraestruturas computacionais baseada na conectividade e no intercâmbio de dados. De diferentes modos, as plataformas tornam mensuráveis diversas práticas sociais, contribuindo decisivamente para a consolidação da datificação como forma hegemônica de conhecimento (van Dijck, 2017).

A pretensão de transformar tudo em dados armazenáveis e, a partir deles, gerir diversos processos de monitoramento, ranqueamento e predição é uma característica central da datificação. Uma das consequências da adoção generalizada deste modelo científico é um avanço da ideologia do dataísmo, ou seja, de uma "crença generalizada na quantificação objetiva" (van Dijck, 2017, p. 43) propiciada pelos dados ${ }^{8}$. No campo das pesquisas acadêmicas que se apropriam de dados obtidos via APIs, a ênfase excessiva no volume de dados coletados,
${ }^{7}$ No original: "a platform is fueled by data, automated and organized through algorithms and interfaces, formalized through ownership relations driven by business models, and governed through user agreements.

\footnotetext{
${ }^{8}$ Esta perspectiva crítica se ancora em um conjunto de discussões que, desde o início da década de 2010, vêm enfatizando que não há objetividade ou neutralidade nas iniciativas de armazenar, interpretar e gerir o social a partir de dados estruturados - ver, por exemplo, as críticas de boyd e Crawford (2012) ao termo "big data".
} 


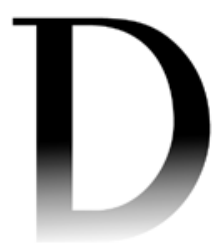

${ }^{9}$ A crítica aqui empreendida a práticas de monitoramento de redes sociais deve-se à premissa de que a disponibilidade de dados de mídias sociais é capaz de fazer emergir um termômetro capaz de medir ou mesmo interpretar debates públicos em tempo real. No mesmo sentido, criticamos estudos de análise de sentimentos que tomam como premissa a possibilidade de classificar ou prever emoções ou outras subjetividades através do processamento de dados sobre este item, ver van de Ven

(2017).

a pouca transparência dos processos metodológicos empreendidos e a adoção indiscriminada de termos como monitoramento ou análise de sentimentos ${ }^{9}$ podem ser indícios de uma excessiva crença na representatividade dos resultados obtidos.

Recorrendo aos Estudos de Plataforma, apontamos aqui que o processo de datificação deve ser compreendido, de forma indissociável, das extensas redes sociotécnicas nas quais a produção e a circulação de dados se inserem. Retomando trabalho anterior (d’Andréa, 2020), listamos cinco dimensões analíticas a serem consideradas. O processo de datificação nas plataformas online, por exemplo, não pode ser apartado dos desempenhos dos algoritmos de seleção, recomendação ou predição. É fundamental também atentar para a dimensão infraestrutural das plataformas, ou melhor, para os diferentes modos como as plataformas vêm, progressivamente, se constituindo a partir da centralização e do controle de operações e fluxos informacionais que envolvem variados parceiros e usuários finais. $\mathrm{O}$ investimento em sistemas operacionais, sistemas de desenvolvimento de software (SDK), lojas de aplicativos e mesmo cabos submarinos são algumas das iniciativas que ilustram o quão articulados são os processos de plataformização e infraestruturização (Platin et al., 2018).

A rearticulação das relações comerciais de variados setores a partir dos intercâmbios de dados indica a íntima relação do processo de datificação com os modelos de negócio desenvolvidos pelas plataformas online. O valor de empresas como Google e Facebook está diretamente associado à sua capacidade de ofertar serviços baseados em dados, como a publicidade direcionada. Pelo volume e complexidade das práticas que abrigam, as plataformas se veem obrigadas a elaborar diferentes mecanismos de governança (Gorwa, 2019). Este processo de autorregulação é implementado, gerido por meio de regras, recomendações e outros recursos normativos e está diretamente relacionado com as políticas de acesso aos dados via APIs, como discutiremos à frente. Por fim, é fundamental atentar para as affordances das plataformas, isto é, para os usos possíveis das funcionalidades que condicionam as ações (Bucher \& Helmond, 2018). A partir das negociações estabelecidas pelos diferentes tipos de usuários com as materialidades disponíveis, pode-se compreender melhor os usos e as práticas associadas às APIs e outros instrumentos de datificação.

De forma complementar à perspectiva analítica multifacetada elaborada no âmbito dos Estudos de Plataformas, um conjunto de proposições e experimentações metodológicas também fundamentado no campo STS busca compreender como as dinâmicas comunicacionais das plataformas podem ser estudadas em articulação com suas materialidades, suas escolhas políticas e suas estratégias econômicas. Possivelmente a vertente que melhor sintetiza os esforços de estudar as singularidades e transformações das plataformas seja a dos métodos digitais. 
Em obra que inaugura esta perspectiva, Rogers (2013) aponta para a importância de "seguir os meios", isto é, de desenvolver olhares analíticos e procedimentos metodológicos sensíveis às lógicas que singularizam cada plataforma através de seus mecanismos de busca, modelos de ranqueamento e outros recursos online.

A complexificação das plataformas e o amadurecimento das pesquisas ancoradas nas especificidades dos meios culminou na proposição de perspectivas complementares à dos métodos digitais, como "métodos de interface" (Marres \& Gerlitz, 2016), "mapeamento de questões problemáticas" (em inglês, issue mapping) (d'Andréa \& Melgaço, 2019; Marres, 2015) e "métodos programados" (Borra \& Rieder, 2014). Vale esclarecer que, embora parte significativa das pesquisas empíricas, ferramentas e protocolos de pesquisa vinculados aos métodos digitais se baseiem na apropriação de dados fornecidos pelas APIs das plataformas, esta perspectiva não se limita à vertente nomeada por Venturini e Rogers (2019) como "pesquisas baseadas em APIs"10. De modo complementar, nem toda pesquisa baseada em métodos computacionais de coleta e tratamento de dados seria, conforme a perspectiva de Rogers e colegas, alinhada à proposta dos métodos digitais.

Em publicação mais recente, Rogers (2018b) ressalta que o que singulariza a perspectiva dos métodos digitais é se apropriar, "para outros propósitos", dos dados obtidos junto às plataformas, o que deve levar as pesquisas a tensionar as lógicas hegemônicas adotadas pelas empresas. Já Gerlitz e Rieder (2018), preocupados com os fluxos descentralizados de dados geridos pelas plataformas, argumentam por uma "especificidade do meio que não é reduzida a um entendimento único e monolítico do que significa um recurso do meio, mas considera os dados digitais como resultados e traços de realizações distribuídas"11 (p. 530).

O ponto sensível que gostaríamos de ressaltar é a preocupação de posicionar os procedimentos metodológicos não como operações instrumentais que tornam visíveis uma realidade que existe a priori, mas como procedimentos que, a partir das escolhas possíveis, fazem emergir leituras e visões de mundo indissociáveis dos gestos políticos e materiais dos vários atores envolvidos. Esta abordagem adotada pelos métodos digitais e propostas afins alinha-se a discussões anteriores em torno da vida social dos métodos, que, no campo STS, são entendidos como um "redes de práticas performativas e materialmente complexas"12 (Law, 2017, p. 47) que articulam entidades heterogêneas, como sujeitos, objetos, imaginários, normas e instituições. Nesse sentido, destacamos a importância de se compreender os procedimentos metodológicos das pesquisas baseadas em dados de APIs como parte de políticas e de práticas incorporadas anteriormente pelas plataformas e, ao mesmo tempo, como articuladores de formas de conhecimento que dependem desses procedimentos para existir.
${ }^{10}$ Uma notória exceção é o uso de crawlers para mapear redes de hiperlinks entre websites (Rogers, 2013). Uma iniciativa recente de operacionalização desta perspectiva é a ferramenta Hyphe (https:// hyphe.medialab.sciences-po. $\mathrm{fr} /$ )

${ }^{11}$ No original: "specificity that is not reduced to a single, monolithic understanding of what a medium feature stands for, but considers digital data as outcomes and traces of distributed accomplishments materially complex and performative webs of practice".

${ }^{12}$ No original: "materially complex and performative webs of practice". 


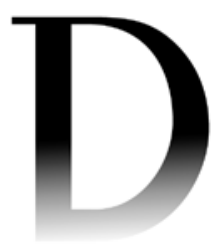

${ }^{3}$ REST é a sigla em inglês para representational state transfer. Proposto no ano 2000, permite transferências direcionadas de recursos específicos da web, que são identificados através de uniform resource identifiers (URI). A arquitetura REST viabiliza as requisições de dados feitas por uma parte significativa das API.

${ }^{14}$ No original: "reformat the web according to the logic of social media".

\section{(POLÍTICA DAS) APIS: PRIMEIRAS INICIATIVAS}

No âmbito das plataformas online, a centralidade do processo de datificação deve ser entendida a partir do desenvolvimento de padrões e de protocolos próprios de intercâmbio de dados. Na virada dos anos 2000, a adoção de arquiteturas de softwares orientadas a recursos da web (estilo REST) ${ }^{13}$ se tornou viável a partir de ampliação de parcerias com desenvolvedores externos, o que culminou no processo nomeado por Helmond (2015) como plataformização da web, ou seja, a consolidação da plataforma como um modelo infraestrutural e econômico que "reforma a web de acordo com a lógica das mídias sociais"14 (p. 2). Segundo a autora, esta mudança se ancora em três precondições de programabilidade: a separação de conteúdo e apresentação (formato XML); a modularização de conteúdo e recursos (permitindo, por exemplo, que vídeos e postagens fossem incorporados a páginas externas); e a interface com bancos de dados.

Em comum, estas inovações permitiram o desenvolvimento de serviços e modelos de negócio baseados na expansão dos serviços online para além de seus websites. $\mathrm{O}$ cruzamento de dados providos por dois ou mais serviços (mashups), a adoção de widgets e plug-ins sociais (Gerlitz \& Helmond, 2013) e a possibilidade de fazer postagens cruzadas são algumas das primeiras aplicações desenvolvidas pelas plataformas. A ênfase, cada vez mais, passou a ser na conectividade (van Dijck, 2013) entre sistemas, que passaram a operar em uma dupla lógica: a descentralização de funcionalidades e a recentralização de dados padronizados.

Por serem os "recursos fronteiriços" (Helmond et al., 2019) que interligam a entrada e saída de dados, as APIs contribuíram decisivamente para o processo de plataformização da web. Uma primeira geração de aplicações voltadas para páginas web foi adotada no início dos anos 2000 por empresas de comércio eletrônico, como eBay (2000) e Amazon (2002). Em 2004, a adoção de uma APIs pelo serviço de compartilhamento de fotos Flickr inaugurou um intenso processo de adesão dos até então denominados sites de redes sociais. Não por acaso, no ano seguinte o editor Tim O'Reilly chamou a atenção de um público mais amplo para a web como plataforma e para as potencialidades de uma nova geração de serviços por ele batizada de web 2.0, dando início a um novo ciclo de apropriações empresariais de tecnologias retoricamente orientadas para ampliar práticas de colaboração ou participação.

Como aponta Bodle (2011), a busca por novas oportunidades de negócio baseadas na interoperabilidade das APIs está diretamente relacionada ao "estouro da bolha" das empresas .com, na virada dos anos 2000, mas também pode ser associada ao processo antimonopólio movido pelo governo dos Estados Unidos contra a Microsoft. Ao reorganizar as lógicas de intercâmbios de dados, 
a plataformização sinalizou uma suposta abertura das grandes empresas digitais para uma atuação mais descentralizada, alavancando um processo que, por fim, teve o efeito contrário. Assim, podemos tomar as APIs como infraestruturas computacionais que reorganizaram a atuação econômica, as práticas de desenvolvedores e demais usuários e as relações político normativas dos serviços online.

No caso das mídias sociais, alguns marcos e acontecimentos nos ajudam a localizar a migração do modelo de "sites de redes sociais" para "plataformas de mídia social" (Helmond, 2015). Helmond et al. (2019), por exemplo, apontam que "o Facebook se tornou programável"15 (p. 129) a partir da incorporação de desenvolvedores externos através do serviço Facebook Development Platform, lançado em 2006. Em um pioneiro estudo sobre APIs em um viés que não se limita a uma abordagem computacional, Bodle (2011) destaca como, no caso do Facebook, a adoção progressiva de diferentes aplicações ${ }^{16}$ autorizava um acesso muito ampliado aos dados de usuários. Em meio à grande diversidade de apps de terceiros, o autor aponta para uma "avalanche" de games sociais e de testes ofertados para os usuários. Um destes testes - "This is Your Digital Life" estaria no centro da controvérsia protagonizada anos depois pelo Facebook e pela Cambridge Analytica.

A centralidade das APIs na consolidação de uma plataforma é ainda mais evidente no caso do Twitter. A bibliografia sobre o tema (Ahmed et al., 2017) parece ser unânime em reforçar o quão decisivo o modelo de descentralização e recentralização de dados adotado pelo Twitter desde 2006 foi decisivo para a relevância adquirida em meio ao cambiante ecossistema das plataformas online. Puschmann e Burgess (2014), por exemplo, ressaltam a forte associação entre a popularização da Streaming APIs, que permite a coleta de um fluxo de tuítes logo após sua postagem, e o posicionamento empresarial do Twitter como uma "fonte insubstituível de informação em tempo real"17 (p. 47). As diferentes apropriações desses dados por empresas jornalísticas, pesquisas de mercado e por pesquisas acadêmicas levaram o Twitter a ser tomado como um "termômetro" ou um "sensor" de discussões públicas sobre diferentes temas contemporâneos, consolidando metodologias e análises ancoradas na crença generalizada na capacidade explicativa dos dados (van Dijck, 2017).

A centralidade das políticas de gestão de dados para o Twitter ficou ainda mais evidente a partir de 2010, quando a empresa adotou a primeira de uma série de restrições de intercâmbio de dados, o que incluiu o banimento de anúncios gerenciados por terceiros e a possibilidade de compra de dados por intermédio de empresas como o Gnip (que, em 2014, seria adquirida pelo Twitter). Essas restrições estão diretamente associadas a uma mudança no modelo de negócios da plataforma, que passou a se considerar uma "empresa de informação" (van Dijck, 2013).
${ }^{15}$ No original: "Facebook became programmable”.

${ }^{16}$ Além do Facebook Developer (2006), o autor menciona: Facebook Platform (2007), Facebook Connect (2008), Open Stream (2009) e Open Graph with Instant Personalization (2010). Para uma lista mais atualizada, ver Helmond et al. (2019)

${ }^{17}$ No original: "irreplaceable, real-time information source”. 


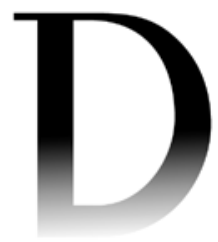

${ }^{18}$ Para uma cronologia introdutória, vale consultar "Histórico das APIs" (s.d.).

${ }^{19}$ Agradecemos ao pesquisador Daniel Loyola pelos insights que ajudaram a aperfeiçoar esta descrição. Eventuais erros factuais são de responsabilidade do autor.

Sem assumir a pretensão de dar conta das nuances históricas das APIs ${ }^{18}$, vale retomarmos brevemente o lançamento algo anedótico da primeira APIs do Twitter, em 2006. Genericamente denominado como “Twitter APIs" (em 2007, seria rebatizada como Twitter REST APIs), o serviço foi brevemente apresentado em um post assinado por um dos fundadores da plataforma (Stone, 2006). Na nota, a "internet" é definida como "séries de tubos", em uma referência a uma expressão usada três meses antes pelo então senador estadunidense Ted Stevens durante um pronunciamento sobre o tema neutralidade da rede. Ao minimizar as atuações comerciais e políticas de provedores de acesso à internet, a fala do senador limitou a internet a uma concepção simplória de infraestrutura e foi repetidamente criticada e ironizada (Schneider, 2006).

O uso desta metáfora por Stone (2006) mostra-se ainda mais curiosa quando observada a imagem que ilustra o post (Figura 1). Trata-se da reprodução de uma estampa de camisa compartilhada originalmente no site Boing Boing (Doctorow, 2006) como parte da "memificação" do bordão do senador Ted Stevens. Na imagem, "Series of Tubes" é associado a objetos que aparentam ser bolsas usadas em hospitais para injetar, em pacientes, soro fisiológico e outras substâncias. Já os rótulos NKX-1, NKX-2 e NKX-3 parecem remeter a proteínas que atuam como reguladoras do desenvolvimento de órgãos do corpo humano ${ }^{19}$.

\section{Figura 1}

Figura usada pelo Twitter na postagem que anuncia o lançamento de sua APIs (2006)

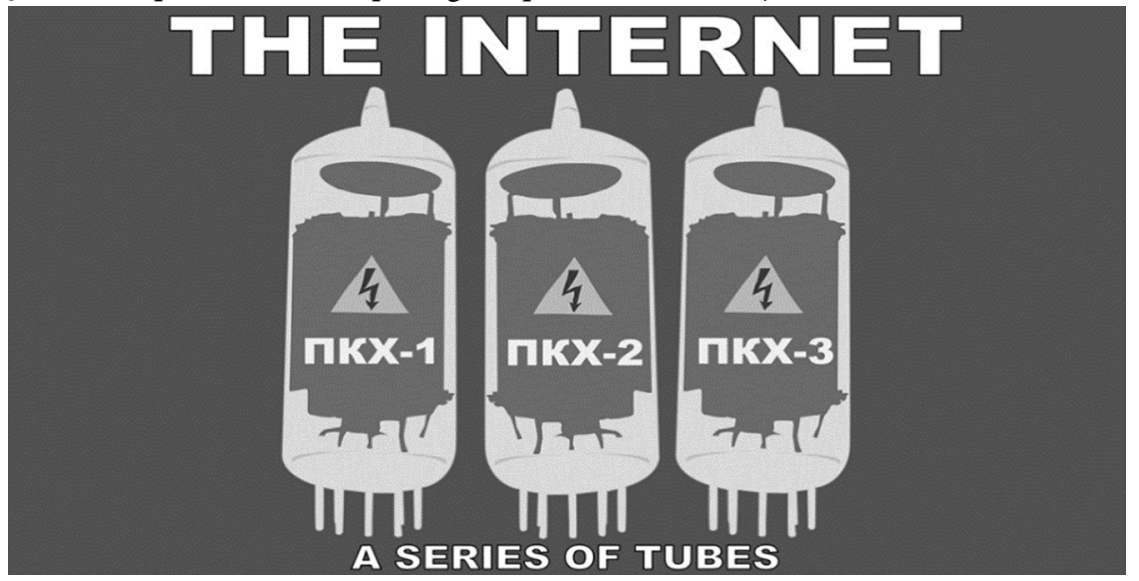

Nota. Stone (2006).

Além de uma referência geek, a postagem que anuncia a primeira APIs do Twitter ilustra como, desde o início, a adoção das APIs esteve intrinsecamente 
ligada a um posicionamento estratégico das plataformas. Apropriando-se da informalidade que, mais à frente, seria reconhecida como parte de uma cultura memética, o fundador do Twitter parece reivindicar para si a capacidade de reinventar a infraestrutura que orientaria a partir de então o fluxo de dados de uma internet interpelada por intensas discussões regulatórias e pela euforia comercial da web 2.0. Neste sentido, a remissão a instrumentos usados em tratamentos médicos pode ser associada a uma pretensa capacidade das APIs de "recuperar" um paciente enfermo (as empresas .com), mas, sobretudo, reforça o argumento de que as aplicações de intercâmbio de dados são construtos tecnocientíficos que produzem conhecimentos através de um processo de "destilação" de dados (Marres \& Weltevrede, 2013).

\section{AFFORDANCES, GOVERNANÇA E MEDIAÇÕES ALGORÍTMICAS}

Operando por meio de um arranjo que envolve infraestruturas, protocolos, regras de uso, interesses econômicos e práticas dos vários tipos de usuários, as APIs colaboram para a conformação de novas leituras de mundo (Bucher, 2013), o que incide decisivamente no modo como devem ser incorporadas às pesquisas que se apropriam de dados obtidos através delas. Partindo das premissas teórico-metodológicas compartilhadas até aqui, detalhamos agora três aspectos centrais relativos à datificação das pesquisas sobre plataformas online: a gramatização das ações, as recentes alterações nas políticas de acesso a dados (governança) e a centralidade dos regimes de visibilidade e hierarquização empreendidos por diversas mediações algorítmicas.

Um primeiro aspecto fundamental para as pesquisas baseadas em APIs é atentar não apenas para quais informações agregadas são fornecidas pela plataforma, mas ainda para as condições com que estas informações foram geradas, organizadas e distribuídas. Deve-se, de antemão, reconhecer o esforço das plataformas em padronizar as atividades dos usuários através de funcionalidades como curtir e compartilhar. Este processo de normatizar o que pode ou não ser feito através das interfaces é chamado por Gerlitz e Rieder (2018) de "gramatização da ação". Usando como exemplo a tecnogramática do Twitter, os autores apontam que formas como tweets, retweets, replies, mentions, ou hashtags "possibilitam às plataformas compactar ... ações, gramáticas e captura de dados, inscrevendo as atividades dos usuários diretamente em unidades muito formalizadas"20 (p. 531).

As APIs, portanto, têm affordances que não só delimitam que dados podem ou não ser acessados por terceiros, mas também predefinem quais e como as ações são inscritas. Gerlitz e Rieder (2018) explicam ainda que
${ }^{20}$ No original: "allows platforms to collapse ... action, grammar, and data capture, inscribing user activities directly into highly formalized units". 


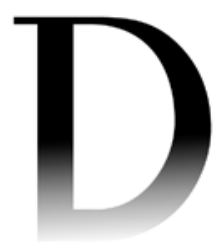

${ }^{21}$ No original: "inputs and outputs".

${ }^{22}$ Uma relação de "ferramentas de pesquisa em mídias sociais" está disponível em Ahmed

(2019).

esta força normativa é visível em quatro momentos articulados: na especificação de entidades e relacionamentos em um banco de dados; nas ações possíveis estabelecidas em infraestruturas (como o back-end e middleware); no controle de "entradas e saídas" exibidos e publicados através de diferentes interfaces (p. 53). Esta multidimensionalidade nos ajuda a compreender como as affordances das APIs estão intimamente articuladas a procedimentos que antecedem e que sucedem as requisições de dados.

Para as pesquisas baseadas em APIs, um aspecto fundamental é lidar com as interpretações e análises induzidas pela gramatização das ações. Dar ênfase à quantidade de curtidas, comentários e compartilhamentos, por exemplo, ajuda a naturalizar leituras baseadas nas lógicas comerciais das plataformas e a naturalizar interpretações do social ancoradas nas noções de popularidade ou engajamento, ou seja, em "métricas de vaidade" (Rogers, 2018a). O resultado, com frequência, são análises excessivamente acríticas e que não tensionam as plataformas. Este risco é ainda mais evidente quando se adere a serviços que se propõem a facilitar o acesso e a sintetizar os dados fornecidos pelas plataformas, como o Netlytic (https://netlytic.org/) ${ }^{22}$. Em versões gratuitas ou pagas, serviços facilitam o processo de interpretação e de análise de dados por meio do empacotamento destes em arquivos como top hashtags, top users, ou co-hashtags, acentuando um processo de encaixapretamento das affordances e outras dimensões das plataformas.

No segundo aspecto fundamental para as pesquisas baseadas em dados, voltamos a atentar para a intrínseca relação entre as políticas das APIs e as questões jurídicas, políticas e econômicas que perpassam a atuação das plataformas. No processo de governança, o que está em jogo é a capacidade das plataformas para propor procedimentos, normas e inovações que possibilitem sua autorregulação em um mercado capitalista global. Conciliar os interesses e demandas de grupos diversos de usuários e parceiros, no entanto, parece ser uma tarefa cada vez mais complicada.

O escândalo envolvendo o uso abusivo de dados obtidos através do Facebook pela empresa Cambridge Analytica pode ser tomado um marco para as discussões em torno da violação de privacidade, do grande poder econômico das plataformas e, por extensão, da governança das APIs públicas. As primeiras revelações sobre o caso vieram a público ainda em dezembro de 2015 e alertavam para o uso de dados obtidos mediante "testes psicológicos" pela campanha do senador estadunidense Ted Cruz à vaga do Partido Republicano para as eleições presidenciais do ano seguinte (Davies, 2015). A eclosão definitiva se deu em maio de 2018, quando revelações feitas pelo 
jornal inglês The Guardian (Cardwallard \& Graham-Harrison, 2018) e outras publicações apontaram que dados de dezenas de milhões de usuários do Facebook teriam sido coletados pela Cambridge Analytica a partir de um teste de personalidade (“This Is Your Digital Life”), que aproveitara a abertura da plataforma para que dados de terceiros fossem também acessados. Ou seja, a política de compartilhamento de dados com terceiros adotada pelo Facebook permitiu não apenas que milhões de cidadãos fossem expostos a anúncios e outras ações hiperpersonalizadas, mas ainda escancarou, para um público mais amplo, como as práticas de colaboração entre usuários da comunidade global presidida por Mark Zuckerberg eram fortemente orientadas para uma otimização do uso comercial da datificação.

Embora o escândalo estivesse claramente associado às práticas comerciais abusivas adotadas, com consentimento do Facebook, por empresas como a Cambridge Analytica, o argumento de proteção à privacidade ganhou centralidade e culminou em consecutivas restrições nos acessos aos dados a partir de APIs públicas. Dentre os marcos deste processo, estão a acelerada restrição dos aplicativos associados à APIs do Instagram (2016), as restrições de acesso aos dados relativos a eventos, grupos e páginas do Facebook (abril de 2018) e a adoção, pelo Twitter, de uma governança mais rígida de seus dados, por exemplo, através da exigência de informações detalhadas sobre os desenvolvedores (junho de 2018).

Para Rogers (2018b), o processo desencadeou "uma crise nas pesquisas sobre mídias sociais" ${ }^{23}$ (p. 558), enquanto Bruns (2019) é mais enfático e afirma tratar-se de uma "APIcalypse”. Fato é que a crescente restrição no acesso aos dados resultou em um significativo rearranjo das pesquisas baseadas em dados e tem desencadeado discussões sobre alternativas às APIs públicas. Freelon (2018), por exemplo, aponta a necessidade de discutirmos a "pesquisa pós-APIs", o que implicaria ao retorno a técnicas de raspagem de dados (scraping) disponíveis na interface das plataformas e em uma melhor compreensão ética e jurídica das consequências das violações dos "termos de uso" das plataformas que, via de regra, proíbem esse tipo de prática.

A pressão por mais transparência levou as plataformas a propor iniciativas institucionais que, em oposição à abertura (ainda que controlada) que guiava as APIs públicas, vêm privilegiando um conjunto restrito e direcionado de pesquisas acadêmicas. O Twitter, por exemplo, lançou uma chamada de proposta de pesquisas que ajudassem "mensurar a saúde" da plataforma. Ao todo 230 propostas foram submetidas e dois projetos foram aprovados ${ }^{24}$. Já o Facebook se aliou a grandes parceiros para lançar, em 2018, o Social Science One, definido pelos coordenadores como "um novo modelo de parcerias entre indústria e
${ }^{23}$ No original: "a crisis in social media research".

${ }^{24}$ Para mais informações, consultar Gadde e Gasca (2018). 


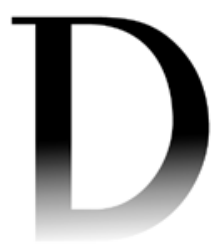

${ }^{25}$ No original: "new model of industry-academic partnerships designed to span this divide among the needs of industry, academia, and the public, even in the most highly politicized environments".

${ }^{26}$ Para mais informações, consultar Shiffman (s.d.).

${ }^{27}$ Para mais informações, consultar King e Persily (2019).

academia, projetado para abranger a divisão entre as necessidades de empresas de tecnologia da internet, pesquisadores acadêmicos e o público, mesmo em ambientes altamente politizados"25 (King \& Persily, 2020, p. 704). Direcionada para pesquisas sobre eleições e democracia, a primeira chamada de propostas anunciou, em abril de 2019, doze projetos contemplados. De acordo com a página do projeto, os grupos de pesquisadores passariam a ter acesso a bilhões de posts e perfis do Facebook e do Instagram (através da ferramenta CrowdTangle e das APIs a ela vinculadas) ${ }^{26}$ e a alguns milhões de anúncios exibidos em diferentes países, incluindo o Brasil (através da Ad Library APIs) ${ }^{27}$. O direcionamento nas temáticas passíveis de serem estudadas é uma das críticas de Bruns (2019) a esta iniciativa.

A terceira e última questão levantada neste artigo diz respeito aos diferentes modos com que os dados coletados através de APIs estão imbricados com as mediações algorítmicas que, entre outras operações, hierarquizam e personalizam os fluxos informacionais nas plataformas. Para tal, é preciso atentarmos, de antemão, para a interdependência entre os conjuntos de dados produzidos, armazenados e (eventualmente) disponibilizados pelas plataformas online e as rotinas computacionais pensadas para produzir relações e hierarquias entre eles. Muitas vezes definido como uma sequência de instruções escritas para cumprir tarefas predeterminadas, os algoritmos são tomados aqui "instâncias que viabilizam uma leitura interessada de uma realidade empírica e 'dataficada” (Rieder, 2018, p. 127).

Frequentemente tomados como opacos, ou como caixas-pretas, os algoritmos assumiram, nos últimos anos, um visível protagonismo nos estudos sobre internet, em especial para discutir fenômenos em torno de polarizações políticas e ampliação das assimetrias de poder. Ao convocar a centralidade das mediações algorítmicas, no entanto, deve-se entender que o que singulariza um algoritmo não são as instruções materializadas em seu código, mas sim o modo como ele articula, na prática, as associações entre um conjunto heterogêneo de atores (Introna, 2016). Isto é, a agência visível dos algoritmos deve ser compreendida em articulação com as infraestruturas que os abrigam, com os mecanismos de governança, com as agências dos usuários e com as demais dimensões das plataformas online.

A partir de suas performances, os algoritmos se relevam como construtos sociotécnicos que instituem novas lógicas de seleção, hierarquização, recomendação e de controle dos fluxos informacionais. Em articulação com as APIs (Bucher, 2013), instauram regimes de conhecimento e de visibilidade que se apropriam dos dados disponíveis para identificar padrões, tendências e, cada vez com mais frequência, para fazer previsões (Bucher, 2018; Gillespie, 2018). Essa 
dinâmica se mostra especialmente central nas "culturas dos rankings" (Rieder et al., 2018) incorporadas pelas plataformas. A hierarquização dos assuntos mais comentados como trending topics e a organização de conteúdos diversos em feeds personalizados são possivelmente as práticas mais evidentes do modo como as plataformas se apropriam dos dados para, em conjunto com as ações de cada usuário, mediar seu uso por meio de algoritmos.

Nosso argumento aqui é que a complexificação dos processos de mediação exercidos pelos algoritmos tem culminado em uma significativa desvinculação entre as lógicas com que as APIs públicas formatam e organizam os dados disponibilizados e as várias experiências possíveis dos usuários nas interfaces das plataformas. As bases de dados coletadas, em geral, induzem uma leitura estática sobre os entrelaçamentos entre uma dada temática e as affordances de uma plataforma, isto é, invisibilizam tanto o modo como uma discussão se modificou ao longo de determinado período de tempo quanto as mudanças nas políticas de governança (critérios para moderação de conteúdo, por exemplo) e em outras dimensões das plataformas. Esta questão é ainda mais evidente quando estamos tratando de um social que, mediado pelas políticas de dados, emerge a partir de métodos que privilegiam uma noção compartilhada de "tempo real" (Marres \& Weltevrede, 2013).

Os desafios impostos pelas mediações algorítmicas às pesquisas sobre plataformas de mídia social mostram-se ainda maiores se atentarmos para como a atuação das plataformas vem sendo orientada por princípios e métodos da inteligência artificial, conforme discute Mackenzie (2018). Para o autor, o funcionamento das plataformas se alinha cada vez menos com o modelo de intercâmbio de dados baseado nas APIs. O centro agora está na programabilidade centrada em processos de aprendizagem de máquina, isto é, em técnicas que se apropriam de dados de treinamento para ajustar e recriar modelos de análise (Mintz, 2019).

Se, no modelo baseado nas APIs, o foco estava na automação do fluxo de dados entre diferentes plataformas, uma política centrada em técnicas de aprendizagem de máquina volta-se para a implementação de workflows e pipelines que permitem que programadores e outros parceiros possam experimentar a implementação e a eficiência de modelos preditivos. Nas palavras de Mackenzie (2018), "a própria plataforma se torna um sistema experimental para observar o mundo e testar como o mundo responde às mudanças na plataforma"28 (p. 2003). Neste modelo, afirma o autor, as plataformas dão um passo adiante para se consolidarem como infraestruturas, colocando em xeque o argumento de van Dijck (2013) sobre a centralidade da "conectividade" para as plataformas online.
${ }^{28}$ No original: "the platform itself becomes an experimental system for observing the world and testing how the world responds to changes in the platform".

MATRIZes 


\section{CONSIDERAÇÕES FINAIS}

Neste artigo, voltamo-nos para um conjunto de questões conceituais e metodológicas a serem enfrentadas por pesquisas baseadas em dados fornecidos através das APIs de plataformas de mídia social. Ancorados nas perspectivas dos Estudos de Plataforma e dos métodos digitais, discutimos como os estudos empíricos baseados em dados devem atentar para as dimensões materiais, políticas, econômicas e normativas que perpassam a atuação de serviços como Twitter e Facebook. De forma cada vez mais evidente, as affordances, as políticas de datificação, a governança, os modelos de negócio e as infraestruturas interferem nas possibilidades e nas restrições impostas às pesquisas empíricas baseadas em dados.

Um primeiro aspecto a ser retomado nestas considerações finais diz respeito tanto às constantes mudanças nos modos de funcionamento das APIs quanto ao papel desempenhado por estes recursos fronteiriços desde o início do processo de plataformização da web, nos anos 2000. Na seção “(Política das) APIs: Primeiras Iniciativas”, retomamos questões relativas ao desenvolvimento de protocolos orientados para um intercâmbio controlado de dados, às pressões jurídicas em torno da crescente concentração de poder por empresas ligadas à internet (telecoms e Microsoft) e à eufórica adesão à proposta "colaborativa" da web 2.0. Mediante exemplos do Twitter e do Facebook, procuramos sintetizar como progressivamente vêm se modificando tanto as estratégias de mercado e de governança das plataformas quanto as políticas das APIs por elas gerenciadas, em uma tendência que complexifica e, em geral, piora as condições de realização de pesquisas empíricas baseadas em dados.

“Affordances, Governança e Mediações Algorítmicas" foram os três aspectos detalhados nessa seção do artigo. Nossa intenção foi, mais uma vez, apontar especificidades que condicionam decisivamente a oferta e as trocas de dados mediadas pelas plataformas. Os esforços das plataformas em padronizar as atividades dos usuários e as implicações de uma adesão acrítica às lógicas de popularidade ou engajamento são pontos a serem observados por metodologias que visem tensionar, e não apenas seguir as affordances das APIs. Já as significativas mudanças na governança de dados após o escândalo Cambridge Analytica sugerem não só uma crise no modelo de pesquisa baseado nas APIs públicas, mas também um aumento na assimetria entre as pesquisas realizadas em países em condições mais precárias de infraestrutura e articulação política com as plataformas. Por fim, procuramos destacar como a centralidade e diversidade das mediações algorítmicas aplicadas aos dados instauram, para cada usuário ou público, regimes diversificados de visibilidade e hierarquização, o que exemplifica a 
acentuação da dimensão experimental das plataformas, principalmente por meio da consolidação da aprendizagem de máquina como modelo prioritário de tratamento de dados.

Cabe, por fim, nos perguntarmos: estaríamos aqui identificando ou insinuando o esgotamento do modelo de APIs públicas adotado (ainda) por algumas plataformas de mídia social? Mais interessados em levantar do que em responder à questão, este artigo procura sinalizar que os dados fornecidos via APIs são sim um rico e potente material empírico se os compreendermos como pistas que sinalizam os modos com o social, os métodos e as plataformas se coproduzem e são interdependentes. Assim, evitar usos instrumentais e positivistas dos rastros fornecidos pelo Twitter, Facebook ou YouTube é, em última instância, não aderir à "ideologia do dataísmo" (van Dijck, 2017), ou seja, evitar que a crença entusiasmada na suposta possibilidade de se revelar o social através dos dados se sobreponha a uma leitura crítica que, além de compreender as dinâmicas das plataformas, busque desnaturalizá-las e tensioná-las. M

\section{REFERÊNCIAS}

Ahmed, W. (2019, 18 de junho). Using Twitter as a data source: An overview of social media research tools. LSE Impact Blog. http://bit.ly/3r14GWP

Ahmed, W., Bath, P. A., \& Demartini, G. (2017). Using Twitter as a data source: An overview of ethical, legal, and methodological challenges. In K. Woodfield (Ed.), The ethics of online research (Vol. 2, pp. 79-107). Emerald. https:// doi.org/10.1108/S2398-601820180000002004

Bodle, R. (2011). Regimes of sharing. Information, Communication \& Society, 14(3), 320-337. https://doi.org/10.1080/1369118X.2010.542825

Borra, E., \& Rieder, B. (2014). Programmed method: Developing a toolset for capturing and analyzing tweets. Aslib Journal of Information Management, 66(3), 262-278. https://doi.org/10.1108/AJIM-09-2013-0094

Boyd, D., \& Crawford, K. (2012). Critical questions for big data. Information, Communication \& Society, 15(5), 662-679. https://doi.org/10.1080/13691 18X.2012.678878

Bruns, A. (2019). After the 'APIcalypse': Social media platforms and their fight against critical scholarly research. Information, Communication \& Society, 1-23. https://doi.org/10.1080/1369118X.2019.1637447

Bucher, T. (2013). Objects of intense feeling: The case of the Twitter APIs. Computational Culture, 3. http://bit.ly/3c0jWPJ

Bucher, T. (2018). If... then: Algorithmic power and politics. Oxford University Press. 
Bucher, T., \& Helmond, A. (2018). The affordances of social media platforms. In J. Burgess, A. Marwick, \& T. Poell (Eds.), The SAGE handbook of social media (pp. 233-253). Sage. http://dx.doi.org/10.4135/9781473984066.n14

Cadwalladr, C., \& Graham-Harrison, E. (2018, 17 de março). Revealed: 50 million Facebook profiles harvested for Cambridge Analytica in major data breach. The Guardian. http://bit.ly/3vyYXLo

d'Andréa, C. F. de B. (2018). Cartografando controvérsias com as plataformas digitais: Apontamentos teórico-metodológicos. Galáxia, (38), 28-39. https:// doi.org/10.1590/1982-2554234208

d'Andréa, C. F. de B. (2020). Pesquisando plataformas online: Conceitos e métodos. Edufba. http://repositorio.ufba.br/ri/handle/ri/32043

d'Andréa, C., \& Melgaço, L. (2019, 11-14 de junho). Tecnologias emergentes: "Questões problemáticas" do VAR tuitadas durante a Copa 2018 [Artigo apresentado]. XVIII Encontro Anual da Compós, Porto Alegre, RS, Brasil. https://bit.ly/3c5mHz9

Davies, H. (2015, 11 de dezembro). Ted Cruz campaign using firm that harvested data on millions of unwitting Facebook users. The Guardian. http:// bit.ly/2OOxmVV

Doctorow, C. (2006, 3 de julho). T-shirt design: “The Internet, a Series of Tubes". Boing Boing. http://bit.ly/3eUgs $2 \mathrm{~N}$

Freelon, D. (2018). Computational research in the post-APIs age. Political Communication, 35(4), 665-668. https://doi.org/10.1080/10584609.2018.1477506

Gadde, V., \& Gasca, D. (2018). Measuring healthy conversation. Blog Twitter. http://bit.ly/3r05Q53

Gerlitz, C., \& Helmond, A. (2013). The like economy: Social buttons and the data-intensive web. New Media \& Society, 15(8), 1348-1365. https://doi. org/10.1177/1461444812472322

Gerlitz, C., \& Rieder, B. (2018). Tweets are not created equal. A platform perspective on social media metrics. International Journal of Communication, 12, 528-547. https://bit.ly/3lty2fm

Gillespie, T. (2010). The politics of "platforms". New Media \& Society, 12(3), 347-364. https://doi.org/10.1177/1461444809342738

Gillespie, T. (2018). A relevância dos algoritmos. Parágrafo, 6(1), 95-121. http:// bit.ly/38VfMq5

Gorwa, R. (2019). What is platform governance? Information, Communication \& Society, 22(6), 854-871. https://doi.org/10.1080/1369118X.2019.1573914

Helmond, A. (2015). The platformization of the web: Making web data platform ready. Social Media + Society, 1(2), 1-11. https://doi. org/10.1177/2056305115603080 
Helmond, A., Nieborg, D. B., \& van der Vlist, F. N. (2019). Facebook's evolution: Development of a platform-as-infrastructure. Internet Histories, 3(2), 123146. https://doi.org/10.1080/24701475.2019.1593667

Histórico das APIs no monitoramento e pesquisa em mídias sociais. (s.d.). IBPAD. http://bit.ly/2Nxpdor

Introna, L. D. (2016). Algorithms, governance, and governmentality: On governing academic writing. Science, Technology, \& Human Values, 41(1), 17-49. https://doi.org/10.1177/0162243915587360

King, G., \& Persily, N. (2019, 28 de abril). First grants announced for independent research on social media's impact on democracy using Facebook data. Social Science One. http://bit.ly/3r42Y74

King, G., \& Persily, N. (2020). A new model for industry-academic partnerships. PS: Political Science \& Politics, 53(4), 703-709. https://doi.org/10.1017/ S1049096519001021

Law, J. (2017). STS as method. In U. Felt, R. Fouché, C. A. Miller, \& L. SmithDoerr (Eds.), The handbook of science and technology studies (pp. 31-57). MIT Press.

Mackenzie, A. (2018). From APIs to AI: Platforms and their opacities. Information, Communication \& Society, 22(13), 1989-2006. https://doi.org/10.1080/13 69118X.2018.1476569

Marres, N. (2015). Why map issues? On controversy analysis as a digital method. Science, Technology \& Human Values, 40(5), 655-686. https://doi. org/10.1177/0162243915574602

Marres, N., \& Gerlitz, C. (2016). Interface methods: Renegotiating relations between digital social research, STS and sociology. The Sociological Review, 64(1), 21-46. https://doi.org/10.1111/1467-954X.12314

Marres, N., \& Weltevrede, E. (2013). Scraping the Social? Issues in live social research. Journal of Cultural Economy, 6(3), 313-335. https://doi.org/10.1 080/17530350.2013.772070

Mintz, A. G. (2019). Visualidades computacionais e a imagem-rede: Reapropriações do aprendizado de máquina para o estudo de imagens em plataformas online [Dissertação de Mestrado, Universidade Federal de Minas Gerais]. Repositório institucional da UFMG. http://bit.ly/2P6j3vP

Plantin, J.-C., Lagoze, C., Edwards, P. N., \& Sandvig, C. (2018). Infrastructure studies meet platform studies in the age of Google and Facebook. New Media \& Society, 20(1), 293-310. https://doi.org/10.1177/1461444816661553

Puschmann, C., \& Burgess, J. (2014). The politics of Twitter data. In K. Weller, A. Bruns, J. Burgess, M. Mahrt, \& C. Puschmann (Eds.), Twitter and society (pp. 43-54). Peter Lang US. 
Recuero, R. (2019, 9 de julho). Mídia social, plataforma digital, site de rede social ou rede social? Não é tudo a mesma coisa? Raquel Recuero. http://bit.ly/3tHK4Vw

Rieder, B. (2018). Examinando uma técnica algorítmica: O classificador de Bayes como uma leitura interessada da realidade. Parágrafo, 6(1), 123-142. https://bit.ly/2PagYih

Rieder, B., Matamoros-Fernández, A., \& Coromina, O. (2018). From ranking algorithms to 'ranking cultures': Investigating the modulation of visibility in YouTube search results. Convergence, 24(1), 50-68. https://doi. org/10.1177/1354856517736982

Rogers, R. (2013). Digital methods. MIT Press.

Rogers, R. (2018a). Otherwise engaged: Social media from vanity metrics to critical analytics. International Journal of Communication, 12, 450-472. https://bit.ly/3vOhqE7

Rogers, R. (2018b). Social media research after the fake news debacle. Partecipazione e Conflitto, 11(2), 557-570. http://doi.org/10.1285/i20356609v11i2p557

Schneider, T. (2006, 11 de julho). Mr. Stevens' wild ride through a "series of tubes". Public Knowledge. http://bit.ly/3r04fw5

Shiffman, N. (s.d.). CrowdTangle for academics and researchers. CrowdTangle. http://bit.ly/3r0FIab

Stone, B. (2006, 20 de setembro). Introducing the Twitter APIs. Blog Twitter. http://bit.ly/3s5PiKm

van de Ven, R. (2017, 25 de janeiro). Choose how you feel; you have seven options. INC Longform. http://bit.ly/2Nv82nh

van Dijck, J. (2013). The culture of connectivity: A critical history of social media. Oxford University Press.

van Dijck, J. (2017). Confiamos nos dados? As implicações da datificação para o monitoramento social. MATRIZes, 11(1), 39-59. https://doi.org/10.11606/ issn.1982-8160.v11i1p39-59

van Dijck, J., Poell, T., \& de Waal, M. (2018). The platform society. Oxford University Press.

Venturini, T., \& Rogers, R. (2019). “APIs-Based Research" or how can digital sociology and journalism studies learn from the Facebook and Cambridge Analytica data breach. Digital Journalism, 7(4), 532-540. https://doi.org/1 $0.1080 / 21670811.2019 .1591927$

Vimieiro, A. C., \& Bargas, J. de K. R. (2019). O uso de dados e métodos digitais nas pesquisas em comunicação. Revista Famecos, 26(2), e32473. https:// doi.org/10.15448/1980-3729.2019.2.32473

Artigo recebido em 24 de outubro de 2020 e aprovado em 8 de março de 2021. 